\title{
Preoperative Nutritional Conditioning: Why, When and How
}

\author{
Birgit Borloni Hendrik Huettner Tobias Schuerholz \\ Department of Anesthesiology and Intensive Care, University Hospital Rostock, Rostock, Germany
}

Keywords

Cancer · Prehabilitation · Nutritional status · Optimization . Surgery

\section{Abstract}

Background: Malnutrition is a frequent finding in patients undergoing cancer surgery, especially in the elderly. The decreased nutritional status leads to increased complications and to delayed recovery after the surgical procedure. While established concepts of enhanced recovery after surgery and rehabilitation aim at improving the patient after surgery, the concept of prehabilitation is targeting the phase before surgery. This multimodal concept incorporates preoperative nutritional support. Summary: Nutritional conditioning targets an increase in the functional reserve preoperatively to optimize recovery in the postoperative period. Routinely, it is combined with an exercise program according to the patient's state. Individualized meal plans help to meet the patient's requirements and should start approximately 4 weeks prior to surgery. An important part of the nutritional conditioning is to guarantee a daily protein intake of at least $1.2 \mathrm{~g} / \mathrm{kg}$ body weight. This may be realized by commercially available whey proteins in particular or milk proteins in general. Key Messages: All specialists involved in the care of cancer patients should recognize the care continuum that starts when the need for surgery is identified and aims at increasing the functional capacity throughout the pre-, peri- and postoperative phase by means of prehabilitation, enhanced recovery programs and rehabilitation.

(c) 2019 S. Karger AG, Basel

\section{Introduction}

The nutritional state of a patient facing the burden of a surgical intervention is one factor which is decisive for the result of the procedure. Imbalance of the caloric intake and a deficit of trace elements may contribute to prolonged ventilation and length of stay on the intensive care unit accompanied by an increase in sepsis episodes and antibiotic therapy [1]. Furthermore, in intensive care the clinical outcome may be influenced by various factors like the quantity and composition of calories and the content of micronutrients and other ingredients to modify the adaptive immune system. According to recently published guidelines, a "personalized" nutrition is targeted [2].

Thus, the need for adequate nutritional support in the perioperative setting cannot be overemphasized. Although guidelines exist dealing with nutritional support and assessment in surgical patients, a national enquiry in the UK showed that only 1 out of 4 older surgical patients underwent nutritional assessment and that malnutrition was detected in approximately $60 \%$ of them $[3,4]$. Within the last two decades growing attention was called to the concept of "prehabilitation." This concept includes the decrease in anxiety, the improvement of exercise and the optimization of nutrition [5]. In a multimodal concept to optimize functional results after surgery, nutritional conditioning is a key aspect to achieve optimal results. This overview will deal with three main questions (why, when and how?) with respect to preoperative nutritional conditioning and the concept of prehabilitation.

\section{KARGER}

() 2019 S. Karger AG, Basel
Tobias Schuerholz, MD, PhD

Department of Anesthesiology and Intensive Care, University Hospital Rostock Schillingallee 35

DE-18057 Rostock (Germany)

E-Mail tobias.schuerholz@med.uni-rostock.de 
Fig. 1. Care continuum in surgical cancer

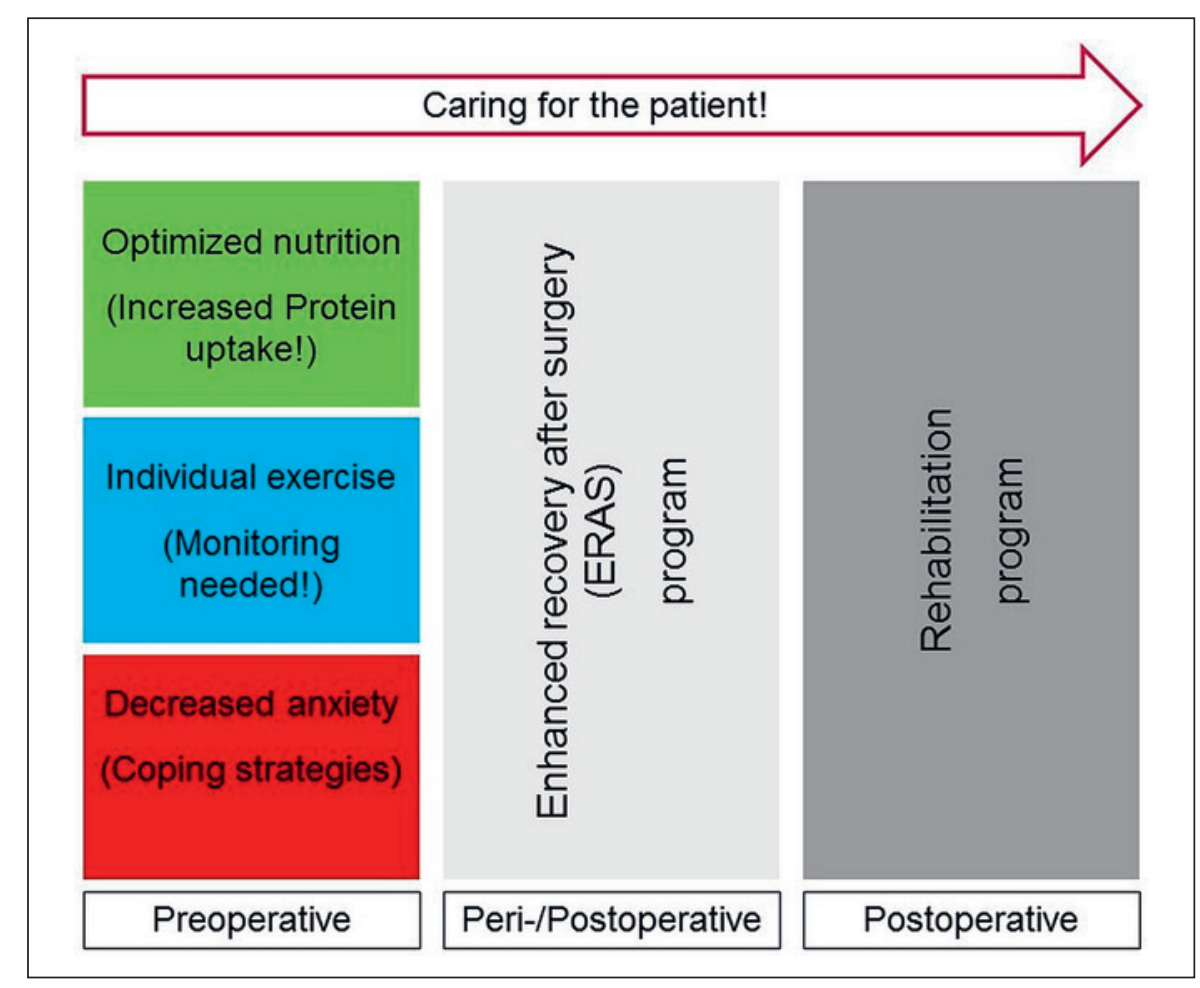
patients.

\section{Why?}

Why should we use preoperative nutritional conditioning?

- Increasing functional capacity

- Augmenting physical strength

- Optimizing postoperative recovery

The underlying reason for the concept of preoperative nutritional conditioning is to increase the functional reserve preoperatively and subsequently to optimize recovery in the postoperative period.

Some studies were based only on increasing the endurance and strength and respiratory muscle training. The PREPARE study failed to prevent the incidence of pneumonia after esophagectomy by a home-based muscle training [6]. The final of the iPEP study (ClinicalTrials. gov identifier: NCT02478996) is scheduled for June 2019 and aims at reducing pulmonary complications during the neoadjuvant therapy before esophagectomy via an Internet-based protocol [7]. A multifaceted approach combining exercise and nutritional support is thought to prepare the patient's condition better than one option alone [5]. Important factors related to inferior outcome are presurgical frailty, age above 75 years and increased worries about the surgical procedure. If these features are taken into account in a multimodal prehabilitation approach, the preoperatively existing functional capacity may be doubled compared to conventional strategies $[5,8]$. Even patients with a markedly reduced preoperative state may increase their health-related quality of life. This leads to augmented physical strength before and after the surgical intervention and a decrease in depressed mental state [9]. In a re-analysis of three studies with 185 colorectal surgery patients in total, Minnella et al. [10] could demonstrate a sustained effect of a prehabilitation program. This was true despite the fact that the prehabilitation group $(n=$ 113) consisted of significantly more patients above 75 years compared to the control group ( 35 vs. $18 \%$; $p=$ 0.012). Furthermore, the self-reported activity at baseline (expressed as the calories needed per kilogram body weight per week in a public health model program) was significantly lower in the prehabilitation group $(22.3 \mathrm{kcal} /$ $\mathrm{kg} /$ week [10.0-46.0] vs. $38.5 \mathrm{kcal} / \mathrm{kg} /$ week (14.2-61.6), $p=0.044$; median [interquartile range]) compared to control. Nevertheless, the included patients with prehabilitation had an increase in 6-min walk distance (6MWD) whereas patients without prehabilitation showed a decrease in $6 \mathrm{MWD}$ after 8 weeks $(+17.0 \pm 84.0$ vs. $8.8 \pm 74.0$ $\mathrm{m} ; p=0.047$ ) [10]. More recently, a randomized clinical trial could confirm these results in 51 patients undergoing surgery due to esophagogastric cancer [11]. Here, the patients in the intervention group were randomized to receive a monitored exercise program 4 times weekly according to their preexisting abilities. Additionally, the protein intake was aimed to guarantee $1.2-1.5 \mathrm{~g} / \mathrm{kg}$ ideal body weight. Both intervention and control group received an enhanced recovery after surgery program as standard of care. The functional capacity reflected by the 6MWD was preserved in the prehabilitation group while control patients showed a loss of functional capacity from 
baseline to the postoperative phase. Moreover, 52\% $(n=$ 12) of the patients with prehabilitation showed improvement in 6MWD compared to $6 \%(n=1)$ in the control group $(p<0.001)$ [11]. A more recent published study protocol aims to achieve an anabolic state by targeting the daily protein intake at $1.5-1.8 \mathrm{~g} / \mathrm{kg}$ body weight. The expected gain in lean body mass is $1-2 \mathrm{~kg}$ [12]. This improved performance is expected to increase functional capacity and to decrease complications throughout surgery. The question why we should perform preoperative nutritional conditioning is answered by the recognition of a disease care continuum that starts when the need for surgery is identified and aims at increasing the functional capacity throughout the pre-, peri- and postoperative phase by means of prehabilitation, enhanced recovery programs and rehabilitation [10]. One main part in this care continuum is the optimization of nutrition (Fig. 1). Alone or in combination with exercise programs, an optimized nutrition may decrease hospital length of stay [13].

\section{When?}

When should we use preoperative nutritional conditioning?

- Include even nonmalnourished patients

- Start support at least 4 weeks before surgery

- Adapt duration to preoperative condition

Although carbohydrate loading (CL) starting the day before surgery was found to be ineffective with respect to length of stay and postoperative fatigue [14], it has been shown that CL in the preoperative phase may halve insulin resistance in the postoperative phase $[15,16]$. This was true for abdominal surgery patients, but these results were not confirmed in studies with patients with coronary artery bypass or with spinal surgery [17]. A further randomized trial in coronary surgery patients described reduced insulin resistance but only transient beneficial effects by enteral CL [18]. Other publications revealed that CL resulted in faster bowel function and retained muscle mass after surgery $[15,19]$. A few years before, it was described that nutritional support is usually applied for 7-14 days and that immune-modulating nutritional support should be initiated 5-7 days preoperatively [3, 20]. In contrast, in the era of beginning prehabilitation the exercise and nutrition programs start between 2 and 12 weeks before surgery. Nevertheless, in many studies the exact time point of the preoperative start of support programs is poorly described.

It is beyond question that patients with a lack of protein energy intake have a higher mortality risk compared to nonmalnourished patients [21]. The muscle mass in critical care patients was shown to be a significant confounder of prognosis especially in the elderly population.
After trauma, sarcopenia led to $32 \%$ mortality compared to $14 \%$ mortality in nonsarcopenic patients $(p=0.018)$ [22]. In general, recent and ongoing studies on prehabilitation are combining exercises adjusted according to the patient's condition and increased uptake of proteins. In some algorithms these interventions are accompanied by measures to decrease anxiety. The above-mentioned iPEP study begins its exercise program 8-12 weeks before surgery. In particular, a personal exercise program for each patient in the iPEP study is based on the functional state at the time of the first diagnosis (approx. 3 months previously). The recorded workout results (e.g., in heart rate) will lead to an adapted training program sent via e-mail in a weekly turnover [7]. Following the precautions others made, a nutritional program should start at the same time. Here, one problem is the varying duration of the support regimen due to differences in the neoadjuvant therapy [11]. The supplement should be consumed after each breakfast or after the exercise program. In some studies, the nutritional support is given even when the patients are not malnourished $[11,23]$. Recently, a nutritional treatment algorithm in Crohn's patients undergoing abdominal surgery has differentiated between lowrisk and high-risk patients [24]. The high-risk patients with severe malnutrition (body mass index $<18.5$, weight loss $10-15 \%$ within 6 months, serum albumin $<30 \mathrm{~g} / \mathrm{L}$ ) should receive exclusive enteral nutrition at least 6 weeks before surgery if $>60 \%$ of energy demand is covered. If at least $60 \%$ of the demand is not achievable, parenteral nutrition should be added [24]. In colorectal cancer, the study intervention started 4 weeks prior to surgery with exercises and whey protein supplement. Compared to patients with rehabilitation, the prehabilitation patients improved their 6MWD before surgery and had higher $6 \mathrm{MWD}$ at 4 and 8 weeks after surgery. Improvement was detected in $58 \%$ of patients with prehabilitation versus $26 \%$ in patients without $(p=0.022)$ [8]. A comparable pilot study in 97 colorectal cancer patients started its intervention of nutritional support 21-46 days before surgery. Prehabilitation resulted in significantly higher 6MWD (prehabilitation $459 \pm 101 \mathrm{~m}$ [mean $\pm \mathrm{SD}$ ] vs. $375 \pm 58 \mathrm{~m}$; $p<0.01)$ and higher self-reported physical activity 2 months after surgery $(p<0.01)$ [25].

A further prehabilitation study protocol shows a start of intervention 4 weeks prior to surgery [12]. The patients will be supplemented by receiving $30 \mathrm{~g}$ whey protein after exercise and before night sleep. The study end is anticipated in October 2019, whereas long-term outcome will be assessed until 1 year after the last patient acquired. Expected results include length of stay, postoperative complications and nutritional status [12]. In lung cancer patients, an exercise program was accompanied for 2-5 weeks by assessment and optimization of a daily diet therapy. The intervention included the daily intake of $6.2 \mathrm{~g}$ of 
branched-chain amino acids [26]. Especially in patients with a higher morbidity (Charlson Comorbidity Index scores $\geq 2$ ), complications were detected in only $27.3 \%$ in the intervention group compared to $68.8 \%$ in conventional treatment $(p=0.0341)$ [26].

According to the recent European Society for Clinical Nutrition and Metabolism (ESPEN) guideline on clinical nutrition in surgery, nutritional support may be indicated in the absence of malnutrition [27]. In "mildly malnourished" patients nutritional support may be applied for up to 10 days. A benefit is seen in cases with "severe metabolic risk," where 10-14 days' nutritional support is advised, even if cancer operations have to be delayed. Whereas outdated guidelines on nutrition only incorporated glucose substitution in the immediate preoperative period, parenteral nutrition was recommended up to 14 days preoperatively in patients with severe malnutrition facing major gastrointestinal surgery [28]. In special patients with infections in the perioperative period a minimum of 6 weeks with nutrition optimization is recommended before reoperation. The "severely malnourished" patient may need longer terms of nutritional support and a combination with an exercise program [27].

\section{How?}

How should we use preoperative nutritional conditioning?

- As part of a multimodal program

- In combination with an exercise program

- Increased protein uptake as important part

The ESPEN formed an oncology expert group that investigated cancer-related malnutrition. They recommended that patients should be supported with individual plans focusing on increased nutrition intake and increased physical activity [29]. Only the intervention by specialists of different subjects working together as shown in some trials will combine knowledge and expertise to increase outcome by multimodal interventions [12].

The introduction of oral nutritional support is considered to be in the first line. This intervention consists of increased meal size, supplements (snacks) and increased nutrient density. When providing nutritional support, it is irrelevant if the patient is at home or in health care or hospital. If a dietitian is advising the patient, it has to be taken into account if shopping and preparing of meals is feasible. A detailed assessment of the patient's capacities will improve the success of the intervention program [30]. In high-risk patients with severe malnutrition, exclusive enteral nutrition should be preferred when $60 \%$ or more of the energy demand may be applied. If this target is not met, parenteral nutrition should be added or total parenteral nutrition may be performed preoperatively and postoperatively [24]. Even obesity is apparently no sign of adequate nutritional status. In an observational trial in 6,518 patients admitted to the intensive care unit, malnutrition more than BMI was a confounder of mortality [31].

Carli et al. [32] could demonstrate in a randomized controlled trial in colorectal surgery patients that intensive physical exercise programs alone did not improve outcome due to low compliance. The concept of a multimodal intervention including nutritional support completed by exercise and anxiety coping gained increasing attention [10-12, 25]. Standardized protein intake in the preoperative phase may contribute to a preferred outcome in several publications. To apply nutritional support registered dietitians provide individual advice according to food diaries completed by the patient for 2 weekdays and 1 weekend day [11]. Patients ought to be in close contact with the dietitians, e.g. by weekly phone calls.

Proteins should be ingested at least every morning and within $1 \mathrm{~h}$ after exercise aimed at $1.2 \mathrm{~g} / \mathrm{kg}$ body weight daily protein intake to increase the muscle gain benefit [33]. A standardized protein meal plan may help to realize the prehabilitation target. Carli et al. proposed a 30 -g protein intake 3 times a day completed by an additional $20 \mathrm{~g}$ of whey protein in a beverage of choice 0-60 min after exercise. This meal plan resulted in approximately $1.5 \mathrm{~g} /$ $\mathrm{kg}$ body weight protein intake per day [5]. The feasibility was demonstrated for commercially available whey proteins in particular and for milk proteins in general $[5,8]$. Whey proteins stimulate protein synthesis in skeletal muscle due to rich leucine content [34]. It may be too early to fall into euphoria because quite a lot of exclusion criteria like contraindications of exercise and/or oral nutrition and disabling diseases exist that prevent patients from benefiting of prehabilitation [11].

\section{Conclusion}

The care of patients in cancer surgery is developing to a care continuum to improve the patient's shape beginning weeks before the surgical procedure. It is a multimodal intervention of different specialists aiming at increasing the patient's physical strength, nutritional status and at decreasing anxiety in view of the procedure. Several studies demonstrated that this approach may lead to improved recovery and outcome after cancer surgery.

\section{Disclosure Statement}

The authors have no conflicts of interest to declare. 


\section{Funding Sources}

The authors declare that there is no funding of any research relevant to the preparation of data or the manuscript.

\section{Author Contributions}

B.B. developed the structure of the manuscript, reviewed the literature, wrote parts of the manuscript and corrected the manuscript thoroughly; H.H. gave substantial implications for the manuscript, created figures and revised the manuscript critically; T.S. wrote the main parts of the manuscript, and made the final structure and corrections of the manuscript.

\section{References}

1 Mayer K. Ernährung. In: Marx G, Muhl E, Zacharowski K, Zeuzem S, editors. Die Intensivmedizin. Berlin, Heidelberg: Springer; 2015. pp. 454-61.

2 Elke G, Hartl WH, Kreymann KG, Adolph M, Felbinger TW, Graf T, et al. DGEM-Leitlinie: Klinische Ernährung in der Intensivmedizin. Akt Ernährungsmed. 2018;43:341-408.

3 Awad S, Lobo DN. What's new in perioperative nutritional support? Curr Opin Anaesthesiol. 2011 Jun;24(3):339-48.

4 Wilkinson K, Martin IC, Giough MJ, Stewart JAD, Lucas SB, Freeth H, Bull B, Mason M. An age old problem. A review of the care received by elderly patients undergoing surgery. London: National Confidential Enquiry into Patient Outcome and Death; 2010.

5 Carli F, Gillis C, Scheede-Bergdahl C. Promoting a culture of prehabilitation for the surgical cancer patient. Acta Oncol. 2017 Feb; 56(2):128-33

6 Valkenet K, Trappenburg JC, Ruurda JP, Guinan EM, Reynolds JV, Nafteux P, et al. Multicentre randomized clinical trial of inspiratory muscle training versus usual care before surgery for oesophageal cancer. Br J Surg. 2018 Apr;105(5):502-11.

7 Pfirrmann D, Tug S, Brosteanu O, Mehdorn M, Busse M, Grimminger PP, et al. Internetbased perioperative exercise program in patients with Barrett's carcinoma scheduled for esophagectomy [iPEP - study] a prospective randomized-controlled trial. BMC Cancer. 2017 Jun;17(1):413.

8 Gillis C, Li C, Lee L, Awasthi R, Augustin B, Gamsa A, et al. Prehabilitation versus rehabilitation: a randomized control trial in patients undergoing colorectal resection for cancer. Anesthesiology. 2014 Nov; 121(5): 937-47.

9 Carli F, Awasthi R, Gillis C, Kassouf W. Optimizing a frail elderly patient for radical cystectomy with a prehabilitation program. Can Urol Assoc J. 2014 Nov;8(11-12):E884-7.

10 Minnella EM, Bousquet-Dion G, Awasthi R, Scheede-Bergdahl C, Carli F. Multimodal prehabilitation improves functional capacity before and after colorectal surgery for cancer: a five-year research experience. Acta Oncol. 2017 Feb;56(2):295-300.

11 Minnella EM, Awasthi R, Loiselle SE, Agnihotram RV, Ferri LE, Carli F. Effect of Exercise and Nutrition Prehabilitation on Functional Capacity in Esophagogastric Cancer Surgery: A Randomized Clinical Trial. JAMA Surg. 2018 Dec;153(12):1081-9.

12 van Rooijen S, Carli F, Dalton S, Thomas G, Bojesen R, Le Guen M, et al. Multimodal pre- habilitation in colorectal cancer patients to improve functional capacity and reduce postoperative complications: the first international randomized controlled trial for multimodal prehabilitation. BMC Cancer. 2019 Jan;19(1):98.

13 Gillis C, Buhler K, Bresee L, Carli F, Gramlich L, Culos-Reed N, Sajobi TT, Fenton TR. Effects of nutritional prehabilitation, with and without exercise, on outcomes of patients who undergo colorectal surgery: a systematic review and meta-analysis. Gastroenterology. 2018;155:391-410. e394.

14 Mathur S, Plank LD, McCall JL, Shapkov P, McIlroy K, Gillanders LK, et al. Randomized controlled trial of preoperative oral carbohydrate treatment in major abdominal surgery. Br J Surg. 2010 Apr;97(4):485-94.

15 Bilku DK, Dennison AR, Hall TC, Metcalfe MS, Garcea G. Role of preoperative carbohydrate loading: a systematic review. Ann R Coll Surg Engl. 2014 Jan;96(1):15-22.

16 Perrone F, da-Silva-Filho AC, Adôrno IF, Anabuki NT, Leal FS, Colombo T, et al. Effects of preoperative feeding with a whey protein plus carbohydrate drink on the acute phase response and insulin resistance. A randomized trial. Nutr J. 2011 Jun;10(1):66.

17 Tran S, Wolever TM, Errett LE, Ahn H, Mazer CD, Keith M. Preoperative carbohydrate loading in patients undergoing coronary artery bypass or spinal surgery. Anesth Analg. 2013 Aug;117(2):305-13.

18 Lee B, Soh S, Shim JK, Kim HY, Lee H, Kwak YL. Evaluation of preoperative oral carbohydrate administration on insulin resistance in off-pump coronary artery bypass patients: A randomised trial. Eur J Anaesthesiol. 2017 Nov:34(11):740-7.

19 Yuill KA, Richardson RA, Davidson HI, Garden OJ, Parks RW. The administration of an oral carbohydrate-containing fluid prior to major elective upper-gastrointestinal surgery preserves skeletal muscle mass postoperatively $-\mathrm{a}$ randomised clinical trial. Clin Nutr. 2005 Feb;24(1):32-7.

20 Weimann A. The surgical/trauma patient. World Rev Nutr Diet. 2013;105:106-15.

21 Mogensen KM, Robinson MK, Casey JD, Gunasekera NS, Moromizato T, Rawn JD, et al. Nutritional Status and Mortality in the Critically Ill. Crit Care Med. 2015 Dec;43(12): 2605-15.

22 Moisey LL, Mourtzakis M, Cotton BA, Premji T, Heyland DK, Wade CE, et al.; Nutrition and Rehabilitation Investigators Consortium (NUTRIC). Skeletal muscle predicts ventilator-free days, ICU-free days, and mortality in elderly ICU patients. Crit Care. 2013 Sep; 17(5):R206.

23 Cederholm T, Bosaeus I, Barazzoni R, Bauer J, Van Gossum A, Klek S, et al. Diagnostic criteria for malnutrition - An ESPEN Consensus Statement. Clin Nutr. 2015 Jun;34(3):335-40.

24 Grass F, Pache B, Martin D, Hahnloser D, Demartines N, Hübner M. Preoperative Nutritional Conditioning of Crohn's PatientsSystematic Review of Current Evidence and Practice. Nutrients. 2017 Jun;9(6):9.

25 Li C, Carli F, Lee L, Charlebois P, Stein B, Liberman AS, et al. Impact of a trimodal prehabilitation program on functional recovery after colorectal cancer surgery: a pilot study. Surg Endosc. 2013 Apr;27(4):1072-82.

26 Harada H, Yamashita Y, Misumi K, Tsubokawa N, Nakao J, Matsutani J, et al. Multidisciplinary team-based approach for comprehensive preoperative pulmonary rehabilitation including intensive nutritional support for lung cancer patients. PLoS One. 2013; 8(3):e59566.

27 Weimann A, Braga M, Carli F, Higashiguchi T, Hübner M, Klek S, et al. ESPEN guideline: clinical nutrition in surgery. Clin Nutr. 2017 Jun;36(3):623-50.

28 Weimann A, Breitenstein S, Breuer JP, Gabor SE, Holland-Kunz S, Kemen M, et al. Klinische Ernährung in der Chirurgie. Akt Ernährungsmed. 2013;38:e155-97.

29 Arends J, Baracos V, Bertz H, Bozzetti F, Calder PC, Deutz NE, et al. ESPEN expert group recommendations for action against cancer-related malnutrition. Clin Nutr. 2017 Oct;36(5):1187-96.

30 Baldwin C. The effectiveness of nutritional interventions in malnutrition and cachexia. Proc Nutr Soc. 2015 Nov;74(4):397-404.

31 Robinson MK, Mogensen KM, Casey JD, McKane CK, Moromizato T, Rawn JD, et al. The relationship among obesity, nutritional status, and mortality in the critically ill. Crit Care Med. 2015 Jan;43(1):87-100.

32 Carli F, Charlebois P, Stein B, Feldman L, Zavorsky G, Kim DJ, et al. Randomized clinical trial of prehabilitation in colorectal surgery. Br J Surg. 2010 Aug;97(8):1187-97.

33 Campbell WW, Leidy HJ. Dietary protein and resistance training effects on muscle and body composition in older persons. J Am Coll Nutr. 2007 Dec;26(6):696S-703S

34 Grey V, Mohammed SR, Smountas AA, Bahlool R, Lands LC. Improved glutathione status in young adult patients with cystic fibrosis supplemented with whey protein. J Cystic Fibrosis. 2003;2:195-98. https://doi. org/10.1016/S1569-1993(03)00097-3. 